\title{
Variations in EEG predict the individual differences in social tasks
}

\author{
Haoming Zhang ${ }^{1}$, Kunkun Zhang ${ }^{2}$, Ziqi Zhang ${ }^{2}$, Mingqi Zhao ${ }^{3}$ \\ Quanying $\mathrm{Liu}^{4}$, Wenbo $\mathrm{Luo}^{2 *}$, Haiyan $\mathrm{Wu}^{1 *}$ \\ ${ }^{1}$ Centre for Cognitive and Brain Sciences and Department of Psychology, \\ University of Macau, Macau, China \\ ${ }^{2}$ Research Center of Brain and Cognitive Neuroscience, \\ Liaoning Normal University, Dalian, China \\ ${ }^{3}$ Research Center for Motor Control and Neuroplasticity, \\ KU Leuven, Leuven, Belgium \\ ${ }^{4}$ Shenzhen Key Laboratory of Smart Healthcare Engineering, \\ Southern University of Science and Technology. \\ *Correspondence email: luowb@lnnu.edu.cn; haiyanwu@um.edu.mo
}

People experience events and form an impression of others in a way that is affected by social influence every day. In the present study, we designed a series of experiments centered on social influence to investigate people's bias in following others' opinions and its underlying neural predictors. Our results showed social conformity and proved that social influence-induced change can be predicted by the amount of electroencephalogram (EEG) variations when people view others' faces. This prediction effect is robust in the alpha-band over the right frontal and left occipital electrodes for negative influence. EEG variations can also predict the subsequent trust difference between negatively and positively influenced faces. Our findings suggest that higher EEG variations in the pre-influence task may serve as a predictor of high vulnerability to social influence. The present study provides a novel approach that considers both the stability of one's endogenous EEG and the variations in external task components to predict human social behaviors. 


\section{Introduction}

Since "beauty is in the eye of the beholder," one's perception or impression of faces is usually subjective (1). This suggests that norms exist in the social perception of faces, which can be highly similar across social contexts. However, previous research has indicated that facial judgment is modulated by various factors, such as personality (2), characteristics of selfresemblance characteristics (3), and culture (4). Therefore, facial judgment is subjective and comprises individual differences. Just as people do not all enjoy the same scenes or like the same characters in a movie, individuals' thoughts and feelings may occasionally deviate from each other, reflecting variations when experiencing social stimuli. These variations in endogenous signals can impact cognitive appraisals of unfolding events and the generation of affective meaning. Consequently, individuals' neural responses may diverge throughout a socially influenced experience, reflecting dynamically shifting alignments of thoughts and feelings. These neural dynamics may reflect the states of stable opinions or feelings that can predict people's behavioral and neural responses when external social information is presented. How can people form a broad impression of others given a combination of these unique endogenous states and external social information?

"Social conformity" refers to people's tendency to adjust to social influence, including others' opinions, choices, and attitudes (5-7). Studies have also suggested that facial evaluation shifts due to social influence in facial judgment experiments $(8,9)$. Further, according to Schnuerch (10), when weaker faces are encoded, the more people conform to the majority response regarding the faces' attractiveness-which could be attributed to the lack of reliable information on the faces. This evidence may indicate that the weaker stable norm one keeps, the more susceptibility to other's opinions. Although evidence is mixed and still developing, prior research suggests that humans are susceptible to peer pressure (11). While it is evident from many psychological studies that human behavior is susceptible to the influence of others $(5,12)$, few attempts have been made to investigate whether neural state stability can predict conformity and the resulting behaviors. In light of the literature showing people's susceptibility to social influences and the valence imbalance in impression formation demonstrated by studies in the field of social psychology (13), we examined whether individuals exhibit individual differences 
in conformity during face perception and whether such differences are predictable from their neural responses (e.g., electroencephalograms [EEGs]) in an independent face task.

Neural signatures from the frontal and occipital visual areas may predict the social influence effect on face perception. Previous studies show the role of the frontal cortex in social conflict detection and resolution $(8,14-16)$. EEG studies have also confirmed that the discrepancy between individual and majority opinions induces error or conflict detection-related components, such as frontal feedback-related negativity (FRN) (10,17-19). For example, research has shown that face evaluation tasks revealed robust frontal negativity when an individual's opinion conflicted with the group's opinion (20). Over the last decades, frontal alpha activities have been associated with affective motivational states, which suggests larger relative left-hemispheric activation during approach-related motivation, and larger relative right-frontal activation during avoidance-related motivation (21-23). In a social influence face perception task, the impression of faces can initially evoke approach or avoidance motivation, and social influence may then elicit conflict signals and aversive states, which can be indicated by frontal EEGs. Furthermore, studies have reported face-selective activation in the occipital cortex (e.g., fusiform face area [FFA]) (24), and a link between facial preference and EEG alpha power during passive viewing and active section tasks (25).

Tracking moment-to-moment variations in neural activity is emerging in neuroscience (26). While it is of potential importance, it has largely been ignored in social behavior prediction. It has been hypothesized that neural variability may reflect a greater dynamic range, such that larger variability is generally beneficial to adaptability and efficiency, which permits a greater range of responses to a greater range of stimuli $(26,27)$. Measures of brain signal variability demonstrate highly predictive results for brain function $(28,29)$. For example, such measures show greater variability during the upright compared to the inverted face condition, especially in the fusiform gyrus and anterior cerebellum (29). A series of studies have shown increased neural variability and lower behavioral variability in development, such that the younger group showed faster and more consistent performances and exhibited higher brain variability across tasks $(27,30,31)$. While most of the previous studies on neural variations are tracked from the resting state, further studies also indicate the predictability of neural activity preceding the task to fluctuations in cognitive flexibility (32). 
In the present study, we focused on alpha-band EEGs during both an independent social situation and the main tasks, given that (i) the significance of alpha band power in emotion or motivation is well understood, especially among resting EEG studies; and (ii) the alpha power in different stages of life is thought to predict various human abilities $(33,34)$. Thus, it may serve as a good indicator of individual differences in brain states. Since no previous study has tried to extract alpha-band EEG in one task to predict behavioral performance or the EEG of subsequent tasks, the novelty of our work stems from the idea that the alpha-band EEG captured in an independent face perception task can predict one's subsequent social perception and decisions. To test our hypothesis, we shall: (1) quantify the EEG variation in a face perception task with stimuli (i.e., sub-experiment 1); and (2) test whether its variation in an EEG can predict the preinfluence to post-influence behavioral change and the decision-making task (sub-experiments 2, 3, and 4) (see Figure 1). Our approach differs from conventional prediction studies in that we make a multiple task design (see Figure 1), and with prior assumptions about the variation characteristic of the EEG, can predict the subsequent mind-changing behaviors. This allows us to consider both the stability of one's endogenous electrophysiological response and the variations in external task components used to predict human decision processes in a social context.

\section{Results}

\section{Behavioral results}

\section{Social influence altered face judgments from pre-influence to post-influence}

Figure 2) presents the behavior change from pre-influence to post-influence under the four conditions. In our analysis, we considered the judgments of the first two repeated faces in Experiment 2 as pre-influence judgments, and the judgments in Experiment 3 (a judgment task without social influence) as post-influence judgments. Behavior change was defined by subtracting the average trust judgment in Experiment 2 (which was scored as 1 if judged as "trust," and scored 0 if judged as "untrust") from the average trust in Experiment 3 for each condition. For condition 0 (where all three peers judged as "untrust"), the average trust rate post-influence was significantly lower than the average trust rate of the pre-influence stage 
$\left(t_{31}=3.44, P=0.0017\right.$ ). For condition 1 (where two peers judged as "untrust", and one peer judged as "trust") ( $\left.t_{31}=0.6897, P=0.4957\right)$ and condition 2 (where one peer judged as "untrust", and two peers judged as "trust") $\left(t_{31}=-0.6574, P=0.5159\right)$, the average trust rate showed no significant difference between pre-influence and post-influence. For condition 3(where all three peers judged as "trust"), the average trust rate after social influence was significantly higher than the average trust rate before social influence $\left(t_{31}=-2.483, P=0.0189\right)$ (see Figure 2).

\section{Trust rate difference between positively and negatively influenced conditions in Experi- ment 4}

For the trust game in Experiment 4, we tested the social influence-induced behavior change, which was defined by subtracting the average trust level in conditions 0 and 1 from the average trust level in conditions 2 and 3 (Figure S1). The paired t-test indicated a significant condition difference $\left(t_{31}=-2.431, P=0.0206\right)$, and that there was a higher trust rate for positive socially influenced faces (conditions 2 and 3 ) than negatively socially influenced faces (condition 0 and 1$)$.

\section{EEG results}

\section{EEG variations in Experiment 1}

In Experiment 1, we first used the EEG signals from all frequency bands. According to the theory on the link between frontal alpha-band power and motivation system (i.e., left and right frontal alpha power reflects approach and withdrawal motivation respectively) (35), we believe that trust and untrust judgments are closely related to motivation directions. Thus, we used a bandpass filter to extract the time-domain EEG epoch signals of alpha bands (8-13 Hz).

To obtain subject-level measures of temporal variation, we first segmented the EEG epoch time series into $\mathrm{m}$ windows, each with a length of $20 \mathrm{~ms}$. Within each time window, the EEG variation was obtained by 1 subtracting the average value of the lower triangular correlation matrix, which was obtained by calculating the Pearson correlations between any two trials in all trials. In Figure 3, we present the correlation matrix of the subjects with the highest vari- 
ation and lowest variation (electrodes AF4 and P7). We show the correlation matrix of all the subjects(electrodes AF4 and P7) in Figure S3 and Figure S4, respectively.

As shown in Figure 4, we observed a general peak variation during 150-200 ms over the right frontal electrode (AF4) and left occipital electrode (P7). Similar patterns can be observed in other electrodes in Figure S9. There was no significant difference in EEG variation between the trust and untrust conditions for electrodes AF4 or P7, so we obtained the mean variation for each participant across the two conditions.

\section{EEG variations in Experiment 1 can predict behavioral changes from pre-influence to post-influence}

After quantifying the EEG variation in Experiment 1, we sought to evaluate whether the EEG variation pattern within one independent face set could predict the following social influence effect in the face perception of another face set. We applied the correlation analysis, using the EEG variations of each electrode in Experiment 1 to predict the behavioral change from pre-influence to post-influence. We also conducted statistical significance tests along with the correlation analysis, and only saved $\operatorname{significant}(P<0.05)$ correlation results. To visualize our results, we plotted the electrodes that were significantly correlated with behavioral changes on a topographic map (see Figure 5A). We found that EEG variation at a large number of electrodes was significantly negatively correlated with behavioral change over a longer period of time in conditions 0 and 1. The negative correlation indicates that the higher the EEG variations in Experiment 1, the more susceptible they are to the negative social influential effect in Experiment 2. For condition 0 (see Figure 5A, upper), the electrodes in the right frontal and left occipital lobe show a significantly negative correlation from $25 \mathrm{~ms}$ to $160 \mathrm{~ms}$. For condition 1 (see Figure $5 \mathrm{~A}$, bottom), the electrodes in the right frontal and left occipital area showed a significantly negative correlation from $35 \mathrm{~ms}$ to $300 \mathrm{~ms}$.

\section{EEG variations in Experiment 1 can predict trust rate in Experiment 4}

Next, we tested whether the EEG variations in Experiment 1 could predict the subsequent social influence effect in Experiment 3. Similar to the previous analysis, we calculated the correlation of EEG variation and behavior difference at each electrode and conducted statistical significance 
tests. We found that the electrodes in the right frontal, right parietal, and left occipital lobe showed a significantly positive correlation from $120 \mathrm{~ms}$ to $280 \mathrm{~ms}$ (see Figure 5B). This positive correlation means that the higher the EEG variations in Experiment 1, the higher the trust rate difference between positively and negatively influenced conditions in Experiment 4.

\section{EEGs under the influence of Experiment 2 can predict behavioral changes from the pre- influence to post-influence stage}

Since the individual EEG revealed that variations can predict behavioral change from preinfluence to post-influence, we asked that whether the EEG variations in others' evaluations would exhibit a predictive effect on subsequent behavioral change? Using the same analysis method, we found that the electrodes over the frontal lobe showed a significantly negative correlation with behavioral changes from $60 \mathrm{~ms}$ to $310 \mathrm{~ms}$ (see Figure 5C). This negative correlation means that the higher the EEG variations under the influence of Experiment 2, the more susceptible they are to negative social influence effects in Experiment 2. Additionally, the correlated map showed a temporal tendency to move from the right frontal lobe to the left frontal lobe. At approximately $110 \mathrm{~ms}$, the right prefrontal lobe showed a significantly negative correlation with behavior; and at $190 \mathrm{~ms}$, the whole prefrontal lobe showed a significantly negative correlation; and at $250 \mathrm{~ms}$, the left prefrontal lobe showed a significantly negative correlation.

\section{EEG variations in Experiments 2 and 3}

After evaluating the temporal EEG variation in Experiment 1, we also evaluated the temporal EEG variation when the participants judged the faces in Experiments 2 and 3. We wondered whether the EEG variations in Experiments 2 and 3 would have the same temporal pattern as the EEG variations in Experiment 1. The method used to calculate the variation of Experiments 2 and 3 was similar to that of Experiment 1. To explore whether the EEG variation would gradually change as the repetition of influence increases, we calculated EEG variations for each condition and each influence step independently.

In previous results, we have found that the region of the right frontal and left occipital lobe was significantly correlated with behavioral changes. To focus on the region of the right frontal and left occipital lobe, we divided the 63 electrodes into six regions of interest (see Figure 
6A) (36). We considered the region 2 as the region of right frontal area, and the region 5 as the region of left occipital area. The variations in each region were calculated by averaging the variations across all electrodes within the given region.

We show the correlation matrix of all the subjects in Experiment 2 (see Figure S5 for region 2, Figure S6 for region 5) and Experiment 3 (see Figure S7 for region 2, Figure S8 for region 5). Additionally, Figure 6B presents the EEG variation when the face set was shown for the first time in Experiment 2, the third time in Experiment 2, and the first time in Experiment 3 as an example. The EEG variations in Experiment 2 and Experiment 3 showed a similar pattern to the EEG variations of Experiment 1. We performed a cluster-level statistical permutation test on the temporal EEG variation of different influence steps and found that there was no significant EEG variation difference as the number of influence steps increased.

\section{EEG variations during the pre-influence period in Experiment 2 can predict behavioral changes from pre-influence to post-influence}

We sought to evaluate whether the EEG variation under the pre-influence face judgment period could predict the subsequent social influence effect. Before we applied the correlation analysis and significance test, we averaged the temporal variations from $0 \mathrm{~s}$ to $300 \mathrm{~ms}$ to obtain the pre-influence mean variations. Next, we used the pre-influence mean variation of each region to predict the behavioral change from pre-influence to post-influence. For condition 0 , we found that the mean variation in region 5 was significantly negatively correlated with behavioral change $(r=-0.415, P=0.02$ ). For condition 1 (see Figure 6C), we found that the mean variation in regions 2 and 5 was significantly negatively correlated with behavioral changes (Region 2: $r=-0.390, P=0.03$; Region 5: $r=-0.467, P=0.008$ ). For conditions 2 and 3 , there was no significant correlation between the mean variation and behavioral change.

\section{Discussion}

Although the understanding of the neurobiological processes associated with social conformity has increased over the years, few studies have attempted to examine whether there is individual differences in neural signals can predict people's sensitivity to social conflict and social con- 
formity. Our study first examined whether the effect of social influence in the face perception task could be predicted by the EEG in a higher variance stimuli task. Therefore, we provide an understanding of how EEG variations can predict social behavior.

\section{Why can EEG variations during high-variance stimuli can predict the so- cial influence effect?}

The human brain processes complex social information in both stable and flexible ways $(37,38)$, integrating speed and efficiency. Even when facing with high variations in external stimuli, a the stable neural system directly processes social information that allows us to generate and maintain a sense of social perception. The fast social brain response is likely higher similar across individuals who share the same social experience $(39,40)$. However, given the same social information, individuals with different social experiences may have unique interpretations, feelings, and decisions-often leading to differed social cognition across individuals $(41,42)$. How can people establish a flexible social functional system that adapts to dynamic social environments, and how can such neural fluctuations in social contexts across individuals independently predict susceptibility to social influence or adjustment under social conflict? Our evidence shows that the stability or variations in specific experiences may be unique to each individual and predict their responses in a social context.

Although it is of considerable importance, how human social behavior can be predicted from task-dependent EEG variations, is still unclear. Primarily, no existing study links pre-task EEG variations to individual differences in subsequent social behavior. The presently used EEG neural distinction between trustworthy and untrustworthy faces may reflect a social perception organizing principle of the brain, which may originate from past social experience or a stable social perception system. Since the decoding accuracy using pattern classification is not significantly higher than the baseline in Experiment 1 (see Figure S2), the EEG signal appears to be more variable across individuals than across conditions. The strong correlation between the EEG variation of Experiment 1 and the EEG variation of pre-influence stage of Experiment 2 further proves this point (see Figure S10). Moreover, the initial distinction between the two types of faces can predict the subsequent rating changes under social influence. Unlike previous EEG alpha power extracted from resting EEG, or or the prediction of single-task performance, 
we provide the first evidence that task-dependent drift for high-variance stimuli can predict social impression changes. That is, the degree to which people are vulnerable to the "disturbance" of peer pressure is predictable from a combination of individual EEG variations.

\section{Prediction effect lasts: EEG in Experiment 1 can predict decisions after social influence}

Studying the stable prediction of the effect is generally involves two ways. One to test the prediction effect across different samples $(43,44)$, and the other way is to test the prediction effect across different time points or tasks $(45,46)$. Neural variability exhibits stability, both across different task conditions, as well as across trials with the same task condition. An recent view proposed shared variability is associated with learning, that "behavioral variability is driven by neural variability" (47). Unlike previous studies that captured EEG features from the resting state, we defined the task-evoked EEG variations to predict subsequent social behaviors, across tasks.

In our findings, the "behavioral variability" induced by the social influence persists to the trust task(higher trust rate for positively influenced faces), even the social influence stimuli is removed in the task. Further,Ithe prediction effect manifested not only on socially influenced changes but also from pre-task EEG variations to trust decisions after the influence task. It showed stable predictability over longer periods, supporting the relative stability of variations. The task evoked behavioral change effect, both the rating change and decision difference to the influenced faces, supports the relative stability of behavioral variability. These prediction effects further support the stable link between behavioral variations and neural variations. These prediction effects of alpha oscillation "bridge the gap" between task-dependent EEGs and taskinduced social behaviors, and provide novel insights into how task-dependent EEGs predict behavior and cognition. They may likewise contribute to the prediction of different aspects of social cognition, such as stereotypes, discrimination, and in-group/out-group effects. 


\section{Specificity of prediction effect: EEG over right frontal and occipital can predict following social behaviors}

EEG oscillations have also been linked to the prediction of human behavior. The alpha frequency between 8 and $12 \mathrm{~Hz}$ was the dominant frequency of the human EEG. In our findings, the prediction effect primarily occurred over the right frontal and occipital areas. Such consistent prediction effect specificity may be interpreted from two accounts: 1) the social task specificity, and 2) the interplay of frontal and occipital areas suggests task-general flexibility and stability in prediction (48).

For the first account, frontal activity is related to reinforcement learning(RL), errors, and conflict processing (see a review by $(49,50)$ ). For instance, in a probabilistic reinforcement learning task, a single-trial analysis revealed that the frontal theta band correlated with reward prediction error (both negative and positive errors) (51). It is also suggested that different prefrontal regions are activated by different types of prediction errors, and the frontal alpha in our results may also reflect this social influence task-dependent activity that can predict the behavioral adjustment after exposure to social information. Since the main task focuses on faces, the prediction effect over the occipital area fits both our hypothesis as well as previous research indicating an association between alpha and the visual representation of the face (52).

On the other hand, since we observed the prediction effect across tasks, we tend to support various task-nonspecific processes. The the prediction alpha activity may also reflect taskgeneral mind wandering $(48,53)$, or a general integration of flexibility and stability in prediction, , as previous research has shown the frontostriatal contribution to the such interplay (54). It is a challenge to give a conclusion on this point given that our tasks are social influence-related processes, resulting in difficulties in generalization to other domains. Further prediction studies on different social or cognitive tasks should be conducted to validate these accounts.

\section{Prediction effect difference across conditions: Higher behavioral changes and predictability for negative social influence conditions}

In the face judgment task, the participants received both positive and negative social influences from their peers. We consistently found a more pronounced effect among negative influence 
conditions, both on behavioral change and EEG prediction. We believe that a prominent explanation for these effects is the negativity bias in social information processing. The behavioral change from Experiment 2 to Experiment 3 (Figure 2) indicated a more pronounced effect and impression updating in the negative influence condition. This supports the presence of a negativity bias that has a greater impact on negative evaluations, compared to available positive evaluations. Positive-negative asymmetry has already been demonstrated in the context of evaluation, attitude, and impression formation (55-58). In an early study on using adjectives to describe another person, participants placed more weight on negative descriptions of others than on positive ones (59). Furthermore, our findings support the theory proposed of Rozin and Royzman (60), who stated regarding negativity dominance, "if we then find that losing $\$ 100$ is as bad as winning $\$ 150$ is good, and that losing $\$ 100$ and winning $\$ 150$ is negative, then we have negativity dominance." Interestingly, participants showed higher predictability for the negative influence condition-indicating that people weigh negative social influence more in the social context. This range to shift to a more negative impression or negativity weight can be better captured or predicted by EEG variations. This further indicates that salient social information processing would be better predicted with task-dependent EEGs.

Overall, the present study provides further evidence that negative information from others has a stronger impact than positive information. It likewise provides the temporal profile of social conflict processing, as revealed by alpha-band EEG and oscillation patterns.

\section{EEGs during social influence predict rating changes}

The reinforcement learning theory of social conformity holds that the differences between outcomes and expectations (i.e., prediction errors) play a crucial role in guiding individual adaptive behavior. Outcomes better than expected reinforce behaviors, while outcomes worse than expected signal a need for adjustment $(61,62)$. Accordingly, the mismatch between one's own evaluation and group ratings may be detected as a "norm prediction error," indicating a need to correct for deviance from norms $(8,14,63,64)$. Following our previous EEG results (65), such error detection signals can be indexed by using the frontal negativity. The present findings further confirm the activation of frontal electrodes in awareness of social conflict or social deviation, which may subsequently predict behavioral change (Figure 5C). Alpha and theta band 
activity has also been well-documented as an oscillation signal associated with awareness and cognitive conflict. Frontal area alpha band activity indicates activity in the anterior cingulate cortex (ACC) (66) and is expected to predict behavioral adjustment. Although we did not examine the alpha band power difference between the negative and positive conditions, we provided further evidence of the predictability of negative influenced conditions. We found that alpha band variation in the Experiment 1, and pre-influence stage of Experiment 2 correlated with the behavioral changes. This indicated that the variation of alpha band neural activity may be associated with the flexibility of social adaptation, which predicts subsequent impression change. This is also evidence of the tuning of one's internal evaluation system toward external social inputs, as the perceived social conflict could predict such integration of a stable personal state and various external social stimuli.

In conclusion, our results provide novel insights into the role of EEG variations in the prediction of social perception and decisions. The present study shows that the prediction effect exists and is more pronounced for more salient information or brain regions, complementing previous research that has implicated EEG indicators in behavior prediction. Our approach is of significance for various social behavior predictions from EEG variation and detects social stability and social flexibility in humans.

\section{Materials and Methods}

\section{Participants}

Thirty-four participants took part in the experiment. Data from three participants were excluded from the analysis due to data loss or EEG artefacts. The remaining 31 participants ( 17 females, 14 males), aged between 19 and 25 years old $(M=21.45, S D=1.959)$, were included in the analysis. There was no difference in the age range for female $(M=21, S D=1.936)$ and male participants $(M=21.818, S D=1.991)$. All participants were right-handed, had normal or corrected-to-normal vision and reported no history of neurological or psychiatric disease. All participants provided written informed consent and were paid 100 Chinese yuan for their participation in the study. 


\section{Face stimuli}

Facial stimuli were obtained from the CAS-PEAL face database (67). First, a total of 160 faces were rated on a seven-point scale (from "1" very untrustworthy to "7" very trustworthy) by 30 participants recruited independently from the formal study. Based on these ratings, we selected two sets of faces that 40 faces for Experiment 1 (face set 1), and another 40 faces for Experiment 2, Experiment 3, and Experiment 4 (face set 2). Specifically, in the face set 1, we included 20 trustworthy faces $(M=5.159, S D=0.443)$ and 20 untrustworthy faces $(M=3.384$, $S D=0.338$ ) for Experiment 1 . The ratings of trustworthy face are significantly higher than the untrustworthy faces $(p<0.01)$. For face set 2 , we selected 40 middle-level trustworthy faces (ratings ranging from 3 to 5). Each face was presented five times in the Experiment 2, twice in the Experiment 3, once in the Experiment 4. The average ratings of the faces in face set 2 were neutral $(M=3.928, S D=0273)$. Notably, the variance and range of trustworthiness in face set 1 are larger than those in face set 2 .

\section{Experiment design and procedure}

The participants sat in a comfortable chair in a dimly lit room facing a computer monitor at a distance of $60 \mathrm{~cm}$. After filling out the informed consent form, the electrode cap was mounted, and the experiment instructions were clearly shown to the participants. They were asked to minimize blinking and maintain visual fixation on a small cross in the center of the screen during the experiments. Each participant performed approximately 10 practice trials before the formal experiments. The full study consisted of four sequential experiments.

\section{Experiment 1: Initial face rating task}

Experiment 1, the initial face rating task, consisted of 40 trials (Figure 1C). Face set 1, which comprised two levels of trustworthiness, was presented in a randomized order in each trial. The participants had to make a binary decision on whether the presented face was untrustworthy (press "F" with the left index finger) or trustworthy (press "J" with the right index finger). Each face is presented for twice in Experiment 1. Behavioral data (e.g., decision time and decisions) and EEG signals were recorded. 


\section{Experiment 2: Social influence task}

Experiment 2 comprised 200 trials (Figure 1D) and consisted of presenting facial set 2 to the participants. Each trial consisted of two rounds of binary decision-making. First, the participants made a binary decision on whether the presented face was untrustworthy (press "F") or trustworthy (press "J"); this was the pre-influence round. The trustworthy levels given by three peers were then presented for $1 \mathrm{~s}$. In the post-influence round, the participant had to make a second decision on the trustworthy level of the face. To illustrate the judgment made by the three other people, three color-coded circles were displayed simultaneously below the face judged by the participant. The circle was displayed in green or red, in order to reflect whether the same face was identified as trustworthy (color $=$ green) or untrustworthy $($ color $=$ red $)$ by other people. . Thus, there were four types of color-coded circle combinations: three red circles, which means all three people rated the face as an untrustworthy face; two red circles and one green circle, which means two people rated it as an untrustworthy face and one rated it as a trustworthy face; one red circle and two green circles, which means one person rated it as antrustworthy face and two rated it as a trustworthy face; and three green circles, which means all three people rated it as a trustworthy face. In this manipulation, there were four conditions (10 faces for each condition), and the participants judged a series of 40 faces five times. In each trial, the participant viewed the screen showing a photograph of a neutral face, and the responses had to be made by pressing one of two keys(untrustworthy face: "F", trustworthy face: "J") with the left and right index finger. Upon responding, the ratings of their peers were shown for $3 \mathrm{~s}$, and the next trial commenced. Note that for each face, the other's rating is kept the same (i.e., each face displays the same three color-coded circles).

\section{Experiment 3: Re-judgements of face set 2}

To test the left-over effects of social influence in Experiment 2, each face was re-judged twice in Experiment 3 (Figure 1E). No social influence was presented in this session. 


\section{Experiment 4: Trust game with face set 2}

To test whether the social influence in Experiment 2 would change the general trustworthiness of the face, rather than only has a rigid memory of the trustworthy level of each face, we design Experiment 4, a multi-round trust game. Experiment 4 consists of 40 trials (Figure 1F), each face in face set 2 was presented once and participants have to choose to invest a certain amount of money to themselves and the presented agent. In each trial, the participant first needs to make investment choices for the agent press "F" represents low investment, and press "J" key represents high Investment. Then, a message will be displayed in the middle of the screen: "You invested $1 \$$ to the agent, and the amount after investment is $3 \$$. Please wait for the agent to allocate the amount...”. After that, the left side of the screen shows the amount the agent repay to the subject, and the right side shows the amount the agent withhold for himself. At the end of each trial, the participants need to rate their happiness of each faces' repayment offer.

\section{EEG data acquisition and preprocessing}

The EEG was continuously recorded from 63 electrodes with BP amplified using the SynAmps system. A common average reference and a forehead ground electrode were used (FCz). Vertical electro-oculographic (EOG) activity was recorded with additional electrodes located below the right eye. For all electrodes the impedance was kept less than $5 \mathrm{k} \Omega$. Electrical activity was amplified with a bandpass of $0.01-100 \mathrm{~Hz}$ and a sampling rate of $1000 \mathrm{~Hz}$. In offline analysis, the data was preprocessed using MATLAB. The data was band-pass filtered between 0.5 to $30 \mathrm{~Hz}$, notched at powerline frequency. Artifacts related to eye and muscle activity were detected and removed by independent component analysis (ICA). Then the data were epoched into single sweep recordings from $700 \mathrm{~ms}$ before to $1500 \mathrm{~ms}$ after stimulus onset. Moreover each epoch was baseline corrected using the signal recorded during $200 \mathrm{~ms}$ that preceded the onset of the stimulus. All epochs with ocular artifacts greater than $30 \mathrm{mV}$ were automatically rejected and in addition were visually scanned to find further artifacts. In the last step, we used bandpass filter to extract time domain EEG epochs signal of alpha bands (8-13 Hz) as the previous theoretic on link between frontal alpha band power and motivation system (35). 


\section{Calculate EEG variations}

To obtain subject-level measures of EEG temporal variation, We used a $20 \mathrm{~ms}$ long time window to segment alpha band EEG epoch time series into $m$ windows with stride equal to $1 \mathrm{~ms}$ (see Figure 7). The time window starts $200 \mathrm{~ms}$ before the stimulus and ends $1000 \mathrm{~ms}$ after the stimulus. Within each time window, we calculated the similarity matrix (see Figure S3) of all trials by Pearson correlation, and averaged the lower triangle of the matrix to obtain the average EEG similarity. Next, average EEG variation is obtained by 1 subtract the average EEG similarity. (see Eq. 1)

$$
V A R_{t, c, s}=1-\overline{\operatorname{corrcoef}\left(S_{i, t, c, s}, S_{j, t, c, s}\right)}, i \leq j=1,2,3, \ldots, n
$$

Where $V A R_{t, c, s}$ denotes the EEG variation in time window $t$, electrode channel $c$, and subject $s$. $S_{i, t, c, s}$ as the EEG signal of $i^{t h}$ trial and window $t$, electrode channel $c$, and subject $s$. $S_{j, t, c, s}$ as the EEG signal of $j^{t h}$ trial and window $t$, electrode channel $c$, and subject $s . n$ as the trial number in total. We have adopted this same measure to address the EEG temporal variation of each subject and electrode.

For the EEG variations when participants judge the faces in Experiment 2 and Experiment 3 , we further divided the 63 electrodes into six regions of interest (see Figure 6A). We allocated the electrodes to six brain regions to pay more attention to the right frontal lobe (region 2) and left occipital lobe (region 5). To obtain more stable spatial patterns, we included the electrodes in the border of two regions in both regions, adapted from (36). The variations in each region were calculated by average variations across all electrodes in this region.

In order to calculate whether the amplitude of each EEG variation is significant relative to baseline (variation $=1$ ), we use one-sample permutation cluster test with a p-value threshold equal to 0.05 , and the number of permutations equal to 10000 . To calculate whether there is significance between the magnitudes of two EEG variations, we use permutation cluster test with a p-value threshold equal to 0.05 , and the number of permutations equal to 10000 . 


\section{Predictive models building}

The main part of our analysis is using the EEG variations to predict the behavioral change(see Figure 7). We calculated the Pearson correlation between EEG variation and behavioral changes of all subjects. We also performed statistical significance tests along with correlation analysis, and retained the correlation results with $P<0.05$.

For EEG variations in Experiment 1 and EEG variations under the influence stage of Experiment 2, we used a $50 \mathrm{~ms}$ long window with a step size of $1 \mathrm{~ms}$ to segment the time series of EEG variations to obtain a more stable temporal correlation pattern. For each time window, we average all points in the time window and use the average value to calculate the correlation with behavioral data. Finally, we get the correlation and p-value between the variation of each electrode at each time point and the behavioral data.

For EEG variations of pre-influence stage in Experiment 2, we select the temporal EEG variations within 0 to $300 \mathrm{~ms}$ for each region. We choose the EEG variations within 0 to 300 $\mathrm{ms}$ for two reasons. The first reason is according to the EEG variation pattern of Experiment 2 (see Figure 6B), the amplitude of variation significantly lower than baseline in this period of time. The second reason is in the results shown in Figure 5, the time period during which EEG variation can be used to predict behavior change is also around $0-300 \mathrm{~ms}$. Next, we average all points in 0 - $300 \mathrm{~ms}$ to a average value and use the average value to calculate the correlation with behavioral data. Finally, we get the correlations and p-values between the variation of each region and the behavioral data.

The corresponding codes for calculating EEG variation are available online at https: //github.com/andlab-um/Trust-untrust-face-judge.

\section{References}

1. J. Hönekopp, Once more: is beauty in the eye of the beholder? relative contributions of private and shared taste to judgments of facial attractiveness. Journal of Experimental Psychology: Human Perception and Performance 32, 199 (2006). 
2. A. C. Little, D. M. Burt, D. I. Perrett, What is good is beautiful: Face preference reflects desired personality. Personality and Individual Differences 41, 1107-1118 (2006).

3. H. Wu, S. Yang, S. Sun, C. Liu, Y.-J. Luo, The male advantage in child facial resemblance detection: Behavioral and erp evidence. Social Neuroscience 8, 555-567 (2013).

4. A. C. Little, D. L. Cohen, B. C. Jones, J. Belsky, Human preferences for facial masculinity change with relationship type and environmental harshness. Behavioral Ecology and Sociobiology 61, 967-973 (2007).

5. R. B. Cialdini, N. J. Goldstein, Social influence: Compliance and conformity. Annu. Rev. Psychol. 55, 591-621 (2004).

6. W. Wood, Attitude change: Persuasion and social influence. Annual review of psychology 51, 539-570 (2000).

7. H. Wu, Y. Luo, C. Feng, Neural signatures of social conformity: A coordinate-based activation likelihood estimation meta-analysis of functional brain imaging studies. Neuroscience \& Biobehavioral Reviews 71, 101-111 (2016).

8. V. Klucharev, K. Hytönen, M. Rijpkema, A. Smidts, G. Fernández, Reinforcement learning signal predicts social conformity. Neuron 61, 140-151 (2009).

9. C. Feng, J. Cao, Y. Li, H. Wu, D. Mobbs, The pursuit of social acceptance: aberrant conformity in social anxiety disorder. Social cognitive and affective neuroscience 13, 809-817 (2018).

10. R. Schnuerch, H. Gibbons, Social proof in the human brain: electrophysiological signatures of agreement and disagreement with the majority. Psychophysiology 52, 1328-1342 (2015).

11. L. Steinberg, K. C. Monahan, Age differences in resistance to peer influence. Developmental psychology 43, 1531 (2007).

12. S. A. Haslam, S. D. Reicher, Contesting the "nature" of conformity: What milgram and zimbardo’s studies really show. PLoS Biology 10, e1001426 (2012). 
13. D. E. Kanouse, L. Hanson, E. E. Jones, H. Kelley, R. Nisbett, S. Valins, B. Weiner, Attribution: Perceiving the causes of behavior. EE Jones, DE, Kanouse, HH, Kelley, RE Nisbett, $S$ Valins, \& B Weiner Eds. Morristown, NJ: General Learning pp. 47-62 (1972).

14. D. K. Campbell-Meiklejohn, D. R. Bach, A. Roepstorff, R. J. Dolan, C. D. Frith, How the opinion of others affects our valuation of objects. Current Biology 20, 1165-1170 (2010).

15. V. Klucharev, M. A. Munneke, A. Smidts, G. Fernández, Downregulation of the posterior medial frontal cortex prevents social conformity. Journal of Neuroscience 31, 11934-11940 (2011).

16. A. Shestakova, J. Rieskamp, S. Tugin, A. Ossadtchi, J. Krutitskaya, V. Klucharev, Electrophysiological precursors of social conformity. Social cognitive and affective neuroscience 8, 756-763 (2013).

17. J. Chen, Y. Wu, G. Tong, X. Guan, X. Zhou, Erp correlates of social conformity in a line judgment task. BMC neuroscience 13, 1-10 (2012).

18. B.-R. Kim, A. Liss, M. Rao, Z. Singer, R. J. Compton, Social deviance activates the brain's error-monitoring system. Cognitive, Affective, \& Behavioral Neuroscience 12, 6573 (2012).

19. R. Schnuerch, S. A. Trautmann-Lengsfeld, M. Bertram, H. Gibbons, Neural sensitivity to social deviance predicts attentive processing of peer-group judgment. Social neuroscience 9, 650-660 (2014).

20. Y. Huang, K. M. Kendrick, R. Yu, Social conflicts elicit an n400-like component. Neuropsychologia $\mathbf{6 5}, 211-220$ (2014).

21. R. J. Davidson, N. A. Fox, Asymmetrical brain activity discriminates between positive and negative affective stimuli in human infants. Science 218, 1235-1237 (1982).

22. J. J. Allen, M. X. Cohen, Deconstructing the "resting" state: exploring the temporal dynamics of frontal alpha asymmetry as an endophenotype for depression. Frontiers in human neuroscience 4, 232 (2010). 
23. D. Metzen, E. Genc, S. Getzmann, M. F. Larra, S. Ocklenburg, Frontal and parietal eeg alpha asymmetry: A large-scale investigation of short-term reliability on distinct eeg systems (2021).

24. G. Yovel, Neural and cognitive face-selective markers: An integrative review. Neuropsychologia 83, 5-13 (2016).

25. J.-H. Kang, S. J. Kim, Y. S. Cho, S.-P. Kim, Modulation of alpha oscillations in the human eeg with facial preference. PloS one 10, e0138153 (2015).

26. D. D. Garrett, G. R. Samanez-Larkin, S. W. MacDonald, U. Lindenberger, A. R. McIntosh, C. L. Grady, Moment-to-moment brain signal variability: a next frontier in human brain mapping? Neuroscience \& Biobehavioral Reviews 37, 610-624 (2013).

27. D. D. Garrett, N. Kovacevic, A. R. McIntosh, C. L. Grady, The modulation of bold variability between cognitive states varies by age and processing speed. Cerebral Cortex $\mathbf{2 3}$, 684-693 (2013).

28. S. W. MacDonald, S.-C. Li, L. Bäckman, Neural underpinnings of within-person variability in cognitive functioning. Psychology and aging 24, 792 (2009).

29. B. Mišic, T. Mills, M. J. Taylor, A. R. McIntosh, Brain noise is task dependent and region specific. Journal of Neurophysiology 104, 2667-2676 (2010).

30. A. R. McIntosh, N. Kovacevic, R. J. Itier, Increased brain signal variability accompanies lower behavioral variability in development. PLoS computational biology 4, e1000106 (2008).

31. D. D. Garrett, N. Kovacevic, A. R. McIntosh, C. L. Grady, The importance of being variable. Journal of Neuroscience 31, 4496-4503 (2011).

32. A. B. Leber, N. B. Turk-Browne, M. M. Chun, Neural predictors of moment-to-moment fluctuations in cognitive flexibility. Proceedings of the National Academy of Sciences 105, 13592-13597 (2008). 
33. J. Obleser, N. Weisz, Suppressed alpha oscillations predict intelligibility of speech and its acoustic details. Cerebral Cortex 22, 2466-2477 (2012).

34. A. Anokhin, F. Vogel, Eeg alpha rhythm frequency and intelligence in normal adults. Intelligence 23, 1-14 (1996).

35. J. V. Honk, D. Schutter, From affective valence to motivational direction: The frontal asymmetry of emotion revised. Psychological Science 17, 963-965 (2006).

36. Yi, Lu, Changming, Wang, Chuansheng, Chen, Gui, Xue, Spatiotemporal neural pattern similarity supports episodic memory. Current Biology (2015).

37. U. Toelch, M. J. Bruce, L. Newson, P. J. Richerson, S. M. Reader, Individual consistency and flexibility in human social information use. Proceedings of the Royal Society B: Biological Sciences 281, 20132864 (2014).

38. M. Kiefer, F. Pulvermüller, Conceptual representations in mind and brain: theoretical developments, current evidence and future directions. Cortex 48, 805-825 (2012).

39. J. M. Lahnakoski, E. Glerean, I. P. Jääskeläinen, J. Hyönä, R. Hari, M. Sams, L. Nummenmaa, Synchronous brain activity across individuals underlies shared psychological perspectives. NeuroImage 100, 316-324 (2014).

40. J. Chen, Y. C. Leong, C. J. Honey, C. H. Yong, K. A. Norman, U. Hasson, Shared memories reveal shared structure in neural activity across individuals. Nature Neuroscience 20, 115125 (2017).

41. R. J. Riding, A. Glass, S. R. Butler, C. W. Pleydell-Pearce, Cognitive style and individual differences in eeg alpha during information processing. Educational Psychology 17, 219234 (1997).

42. M. Nentwich, L. Ai, J. Madsen, Q. K. Telesford, S. Haufe, M. P. Milham, L. C. Parra, Functional connectivity of eeg is subject-specific, associated with phenotype, and different from fmri. NeuroImage 218, 117001 (2020). 
43. M. M. Vandenbosch, D. van't Ent, D. I. Boomsma, A. P. Anokhin, D. J. Smit, Eeg-based age-prediction models as stable and heritable indicators of brain maturational level in children and adolescents. Human brain mapping 40, 1919-1926 (2019).

44. B. Scally, M. R. Burke, D. Bunce, J.-F. Delvenne, Resting-state eeg power and connectivity are associated with alpha peak frequency slowing in healthy aging. Neurobiology of aging 71, 149-155 (2018).

45. J. M. Hausdorff, P. L. Purdon, C.-K. Peng, Z. Ladin, J. Y. Wei, A. L. Goldberger, Fractal dynamics of human gait: stability of long-range correlations in stride interval fluctuations. Journal of applied physiology 80, 1448-1457 (1996).

46. Y. Takagi, J.-i. Hirayama, S. C. Tanaka, State-unspecific patterns of whole-brain functional connectivity from resting and multiple task states predict stable individual traits. NeuroImage 201, 116036 (2019).

47. J. A. Hennig, E. R. Oby, D. M. Losey, A. P. Batista, M. Y. Byron, S. M. Chase, How learning unfolds in the brain: toward an optimization view. Neuron (2021).

48. C. Y. Jin, J. P. Borst, M. K. van Vugt, Predicting task-general mind-wandering with eeg. Cognitive, Affective, \& Behavioral Neuroscience 19, 1059-1073 (2019).

49. J. F. Cavanagh, M. J. Frank, Frontal theta as a mechanism for cognitive control. Trends in cognitive sciences 18, 414-421 (2014).

50. M. X. Cohen, J. F. Cavanagh, Single-trial regression elucidates the role of prefrontal theta oscillations in response conflict. Frontiers in psychology 2, 30 (2011).

51. J. F. Cavanagh, M. J. Frank, T. J. Klein, J. J. Allen, Frontal theta links prediction errors to behavioral adaptation in reinforcement learning. Neuroimage 49, 3198-3209 (2010).

52. M. Miyakoshi, N. Kanayama, T. Iidaka, H. Ohira, Eeg evidence of face-specific visual self-representation. Neuroimage 50, 1666-1675 (2010).

53. C. Braboszcz, A. Delorme, Lost in thoughts: neural markers of low alertness during mind wandering. Neuroimage 54, 3040-3047 (2011). 
54. I. Trempler, A.-M. Schiffer, N. El-Sourani, C. Ahlheim, G. R. Fink, R. I. Schubotz, Frontostriatal contribution to the interplay of flexibility and stability in serial prediction. Journal of cognitive neuroscience 29, 298-309 (2017).

55. M. Blanz, A. Mummendey, S. Otten, Positive-negative asymmetry in social discrimination: The impact of stimulus valence and size and status differentials on intergroup evaluations. British Journal of Social Psychology 34, 409-419 (1995).

56. A. Mummendey, S. Otten, U. Berger, T. Kessler, Positive-negative asymmetry in social discrimination: Valence of evaluation and salience of categorization. Personality and social psychology bulletin 26, 1258-1270 (2000).

57. W. W. Van Dijk, U. Danner, M. Nieweg, S. Sumter, Positive-negative asymmetry in the evaluation of trivial stimuli. The Journal of social psychology 143, 783-784 (2003).

58. G. Peeters, J. Czapinski, Positive-negative asymmetry in evaluations: The distinction between affective and informational negativity effects. European review of social psychology 1, 33-60 (1990).

59. R. S. Wyer, R. L. Hinkle, Informational factors underlying inferences about hypothetical persons. Journal of Personality and Social Psychology 34, 481 (1976).

60. P. Rozin, E. B. Royzman, Negativity bias, negativity dominance, and contagion. Personality and social psychology review 5, 296-320 (2001).

61. C. B. Holroyd, M. G. Coles, The neural basis of human error processing: reinforcement learning, dopamine, and the error-related negativity. Psychological review 109, 679 (2002).

62. W. Schultz, Behavioral theories and the neurophysiology of reward. Annu. Rev. Psychol. 57, 87-115 (2006).

63. P. R. Montague, T. Lohrenz, To detect and correct: norm violations and their enforcement. Neuron 56, 14-18 (2007).

64. E. C. Nook, J. Zaki, Social norms shift behavioral and neural responses to foods. Journal of cognitive neuroscience 27, 1412-1426 (2015). 
65. K. A. Findley, Learning from our mistakes: A criminal justice commission to study wrongful convictions. Cal. WL Rev. 38, 333 (2001).

66. T. Womelsdorf, K. Johnston, M. Vinck, S. Everling, Theta-activity in anterior cingulate cortex predicts task rules and their adjustments following errors. Proceedings of the National Academy of Sciences 107, 5248-5253 (2010).

67. W. Gao, B. Cao, S. Shan, X. Chen, D. Zhou, X. Zhang, D. Zhao, The cas-peal large-scale chinese face database and baseline evaluations. IEEE Transactions on Systems, Man, and Cybernetics-Part A: Systems and Humans 38, 149-161 (2007).

Acknowledgements: The authors thank Dr. Yanyan Qi for helpful comments.

Funding: This study was supported by the SRG from University of Macau(SRG2020-00027ICI ).

Author contributions HW conceived the research. KZ and ZZ conducted the experiment. HZ and HW designed the analyses. HZ, MZ and HW conducted the analyses. All authors wrote the manuscript.

Competing interests The authors declare that they have no competing financial interests.

Data and materials availability: Additional data and materials are available online. 


\section{Figures and Tables}

(A) The structure of experiments

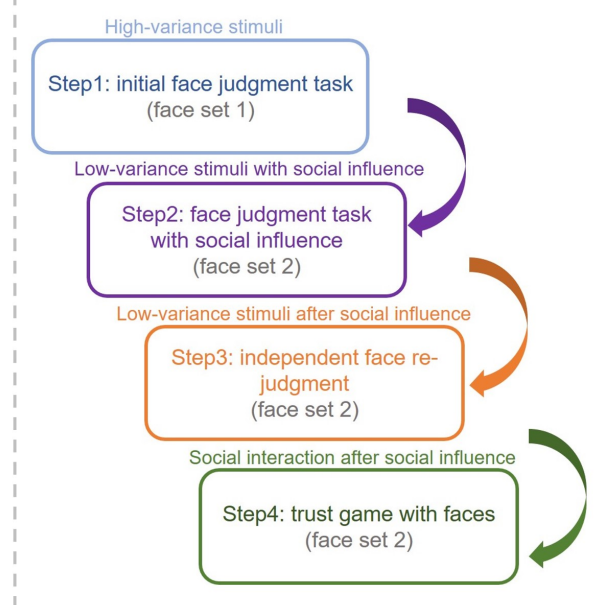

(B) Face stimuli of experiment

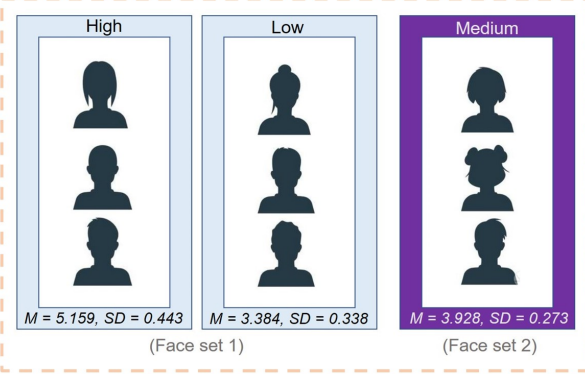

(C) Experimental paradigm of sub-experiment 1

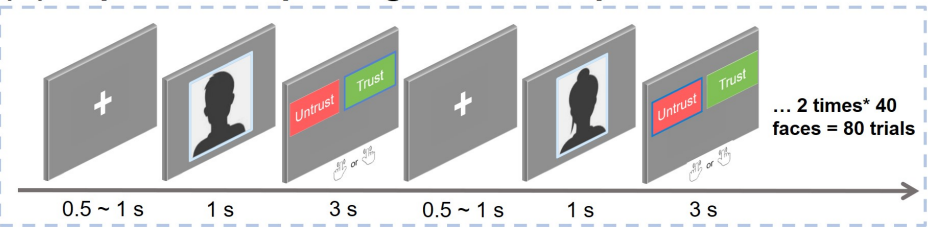

(D) Experimental paradigm of sub-experiment 2

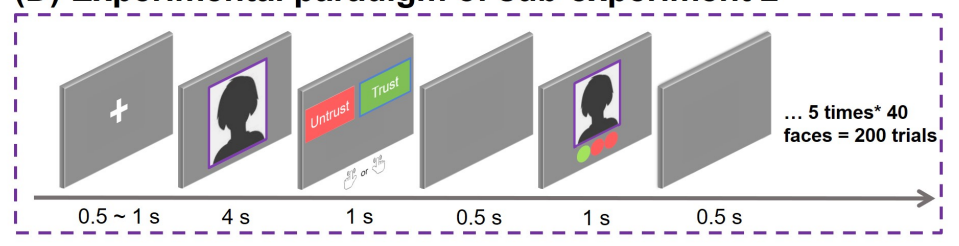

(E) Experimental paradigm of sub-experiment 3

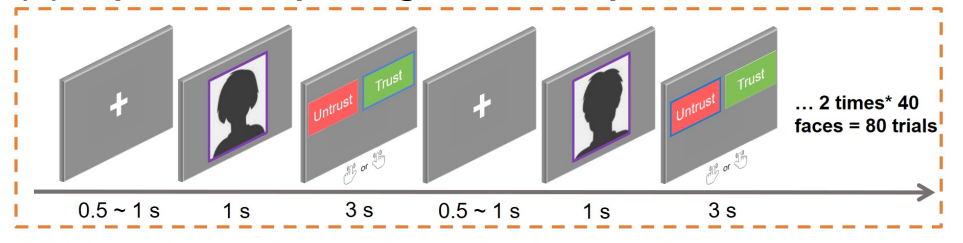

(F) Experimental paradigm of sub-experiment 4

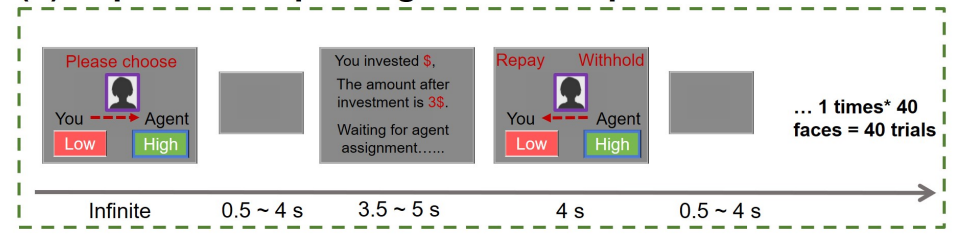

Fig. 1 The experiment paradigm. (A) The structure of the four-step experimental paradigms. All participants needed to conduct four sub-experiments successively. (B) Face stimuli of the experiments. Two sets of face stimuli were selected from a independent trustworthy rating experiment, controlling for some of the edge and contour features of the faces. Based on the trustworthy ratings, our experimental paradigm consisted of two types of facial stimuli, high variance in trustworthy ratings(face set 1), and low variance in trustworthy ratings(face set 2 ). We used face set 1 as facial stimuli in sub-experiment 1 , and used face set 2 in another 3 sub-experiments. (C) Experimental paradigm of sub-experiment 1. The participants need to make binary judgement of whether the presented faces is untrustworthy or trustworthy. (D) Experimental paradigm of sub-experiment 2. Participants were instructed to judge the neutral faces' trustworthiness. After their responses, we will present the judgement from the other three people. (E) Experimental paradigm of sub-experiment 3. Participants would evaluate the faces trustworthiness again which were used in the sub-experiment 2. (F) Experimental paradigm of sub-experiment 4 . Participants were asked to perform a trust-game experiment with each face appeared in sub-experiment 2 and sub-experiment 3 . 

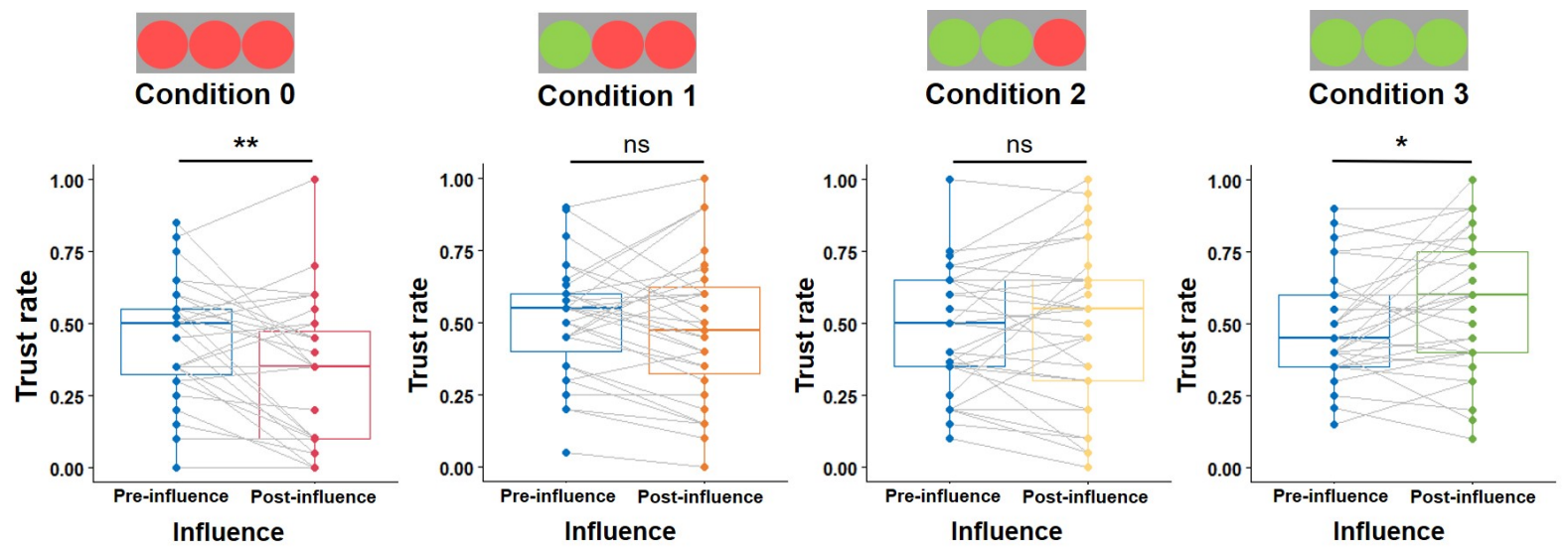

Fig. 2 Behavioral change from pre-influence to post-influence in four conditions. Significance levels (T-test) are indicated by asterisks: $* P \leq 0.05$; $* * P \leq 0.01$; and ns $=$ nonsignificant. Condition 0: all three peers judged as "untrust"; Condition 1: two peers judged as "untrust", one judged as "trust"; Condition 2: one judged as "untrust", two peers judged as "trust"; Condition 3: all three peers judged as "trust".

\section{(A) Similarity matrix of subject 22}

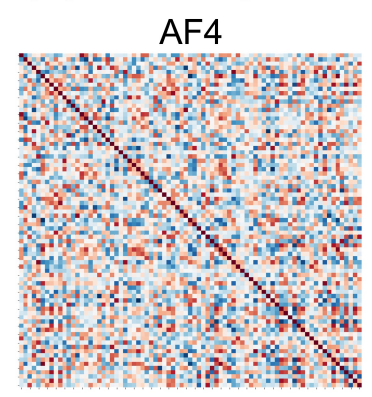

P7

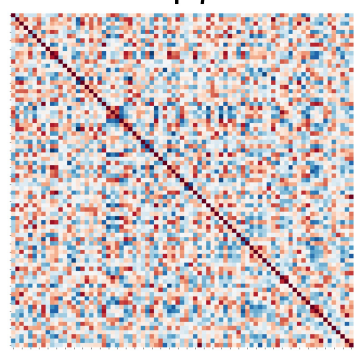

(B) Similarity matrix of subject 33

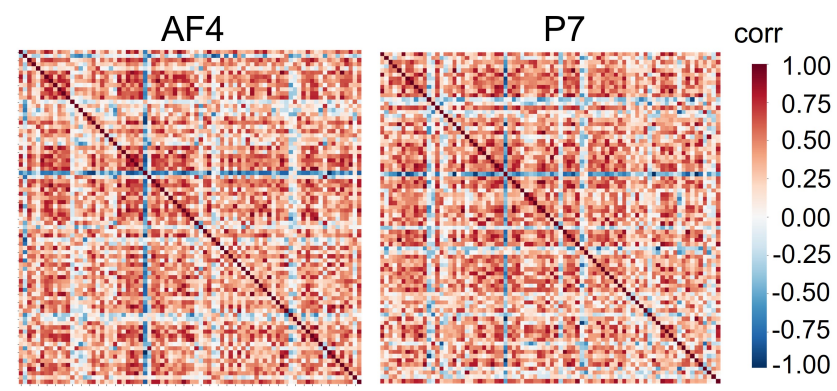

Fig. 3 Some representative similarity matrices calculated using the EEG in Experiment 1.

The similarity matrix was obtained by calculating the Pearson correlation of all trials in pairs, and was averaged between 0-300 ms.. (A) The similarity matrix of the subjects with highest variations (0-300 ms). The similarity matrix was calculated using electrode AF4 (left), and P7 (right). (B) The similarity matrix of the subjects with lowest variations (0-300 ms). The similarity matrix was calculated using electrode AF4 (left), and P7(right). 


\section{(A) Variation of unfiltered EEG}

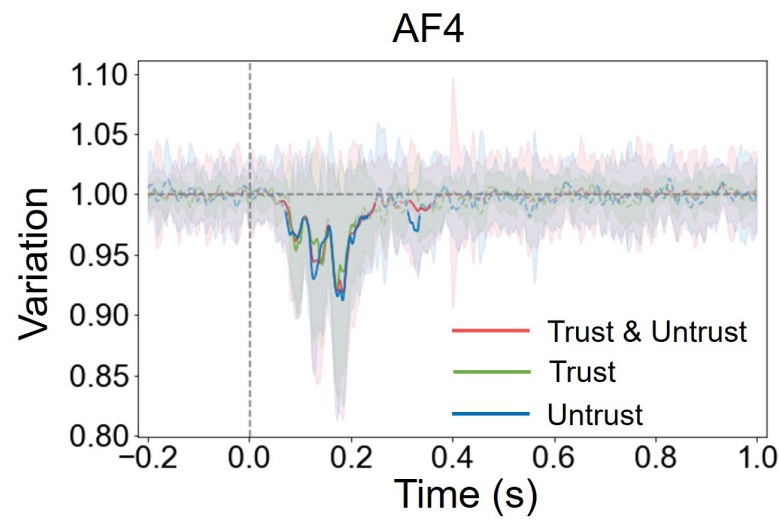

P7

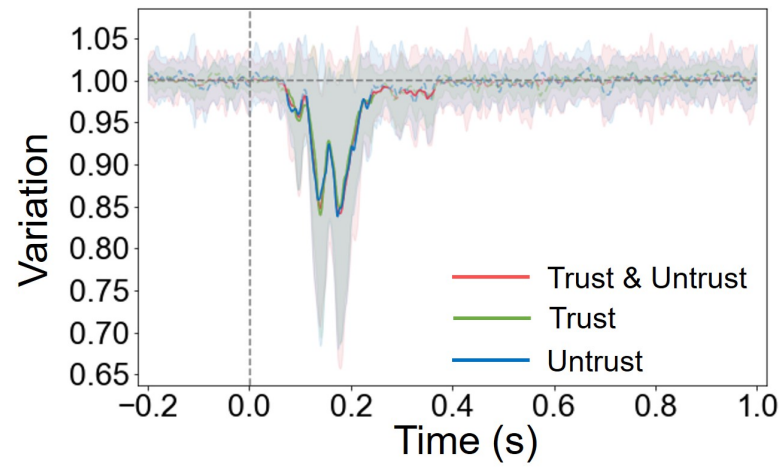

(B) Variation of alpha-band EEG

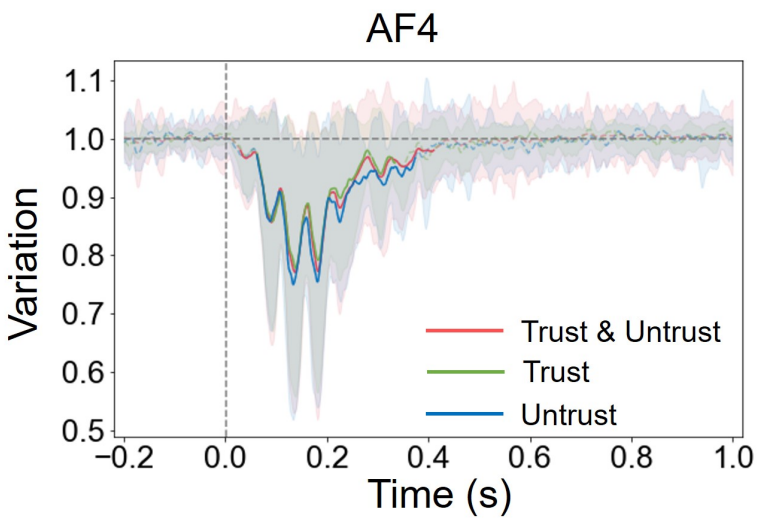

P7

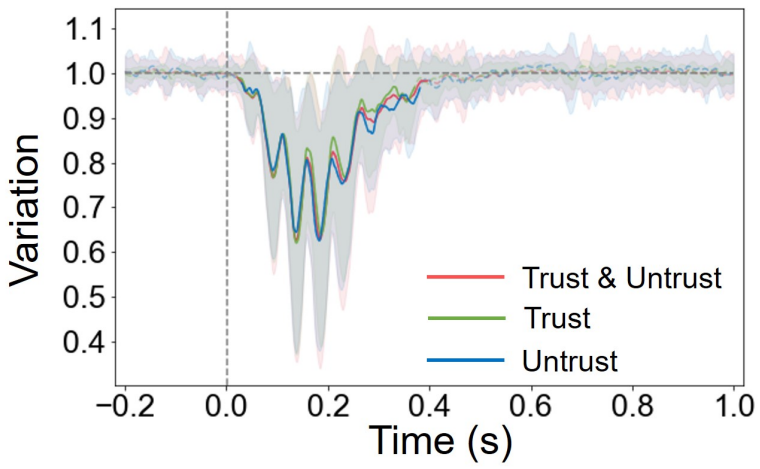

Fig. 4 Some representative epochs of the EEG temporal variation, from electrode AF4 (upper) and electrode P7 (bottom). (A) Variation was calculated using unfiltered EEG. (B) Variation was calculated using alpha band EEG. The red, green, and blue lines are the variation calculated by all events (Trust \& Untrust), only the Trust event and only the Untrust event, respectively. The shaded areas represent the standard deviation. Dashed lines indicate that the variation is not significantly different from the baseline, and solid lines indicate that the variation is significantly different from the baseline. 
(A) EEG in Experiment 1 predicts behavioral change from pre- to post-influence

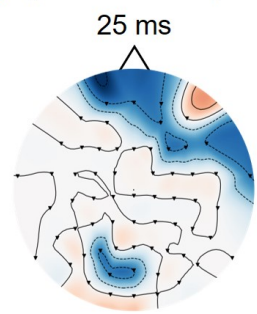

$5 \mathrm{~ms}$

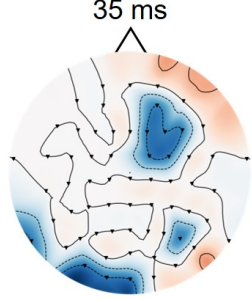

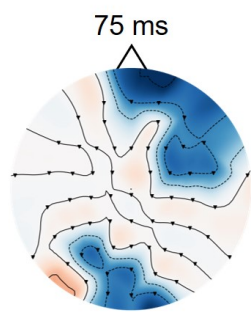

$70 \mathrm{~ms}$

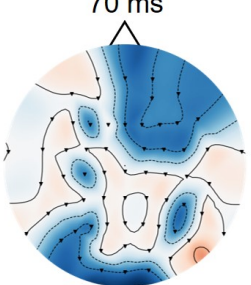

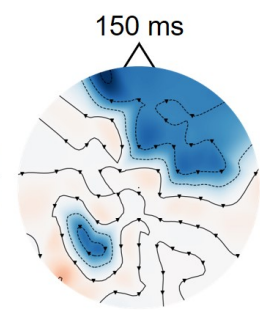
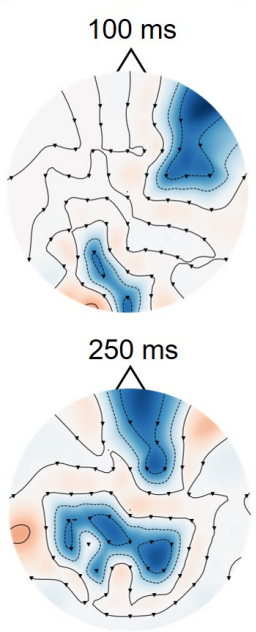
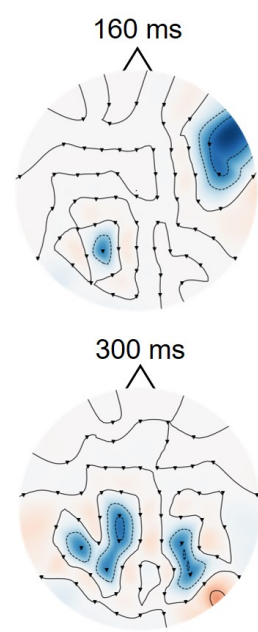

(B) EEG in Experiment 1 predicts behavior in Experiment 4 (condition $2 \& 3-0$ \& 1)
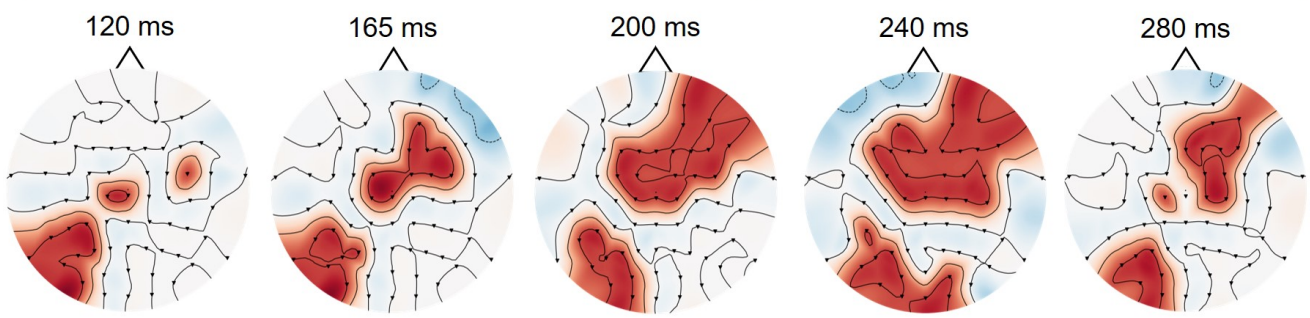

(C) Influence EEG in Experiment 2 predicts behavioral change from pre- to post-influence
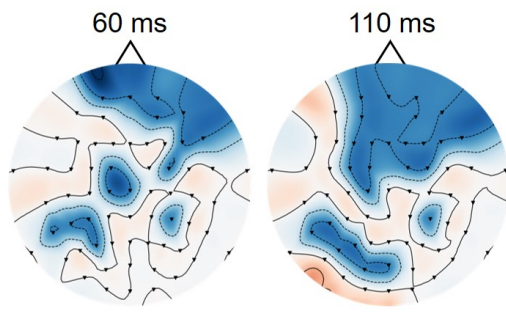

Significantly positive correlation
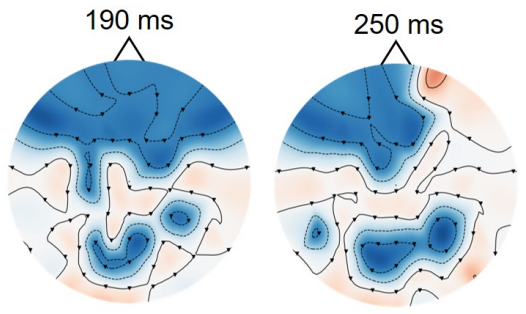

Significantly negative correlation

Fig. 5 The topographic maps shows that the EEG variation in some brain regions can be significantly correlated with behavior. The red and blue areas on the topographic map represent that the EEG variation in these areas are significantly positively and negatively correlated with behavior, respectively. (A) EEG variation in Experiment 1 can predict the behavioral change from pre-influence to post-influence in condition 0 (upper) and condition 1 (bottom). (B) EEG variation in Experiment 1 can predict the difference between conditions 2 and 3 as well as conditions 0 and 1 in Experiment 4. (C) EEG variation under social influence in Experiment 2 can predict the behavioral change from pre-influence to post-influence in condition 1. 
$\begin{array}{lll}\text { (A) EEG electrode in } 6 \text { ROIs } & \text { (C) Pre-influence variation predicts behavioral change }\end{array}$

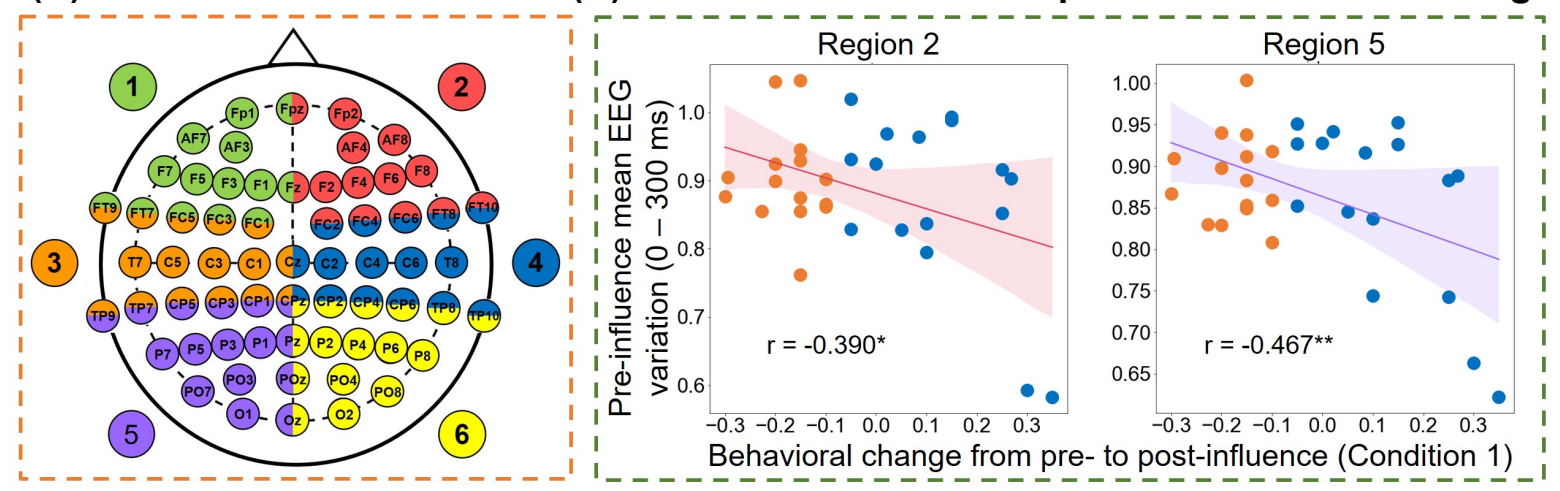

\section{(B) ROI-based alpha-band EEG variation}

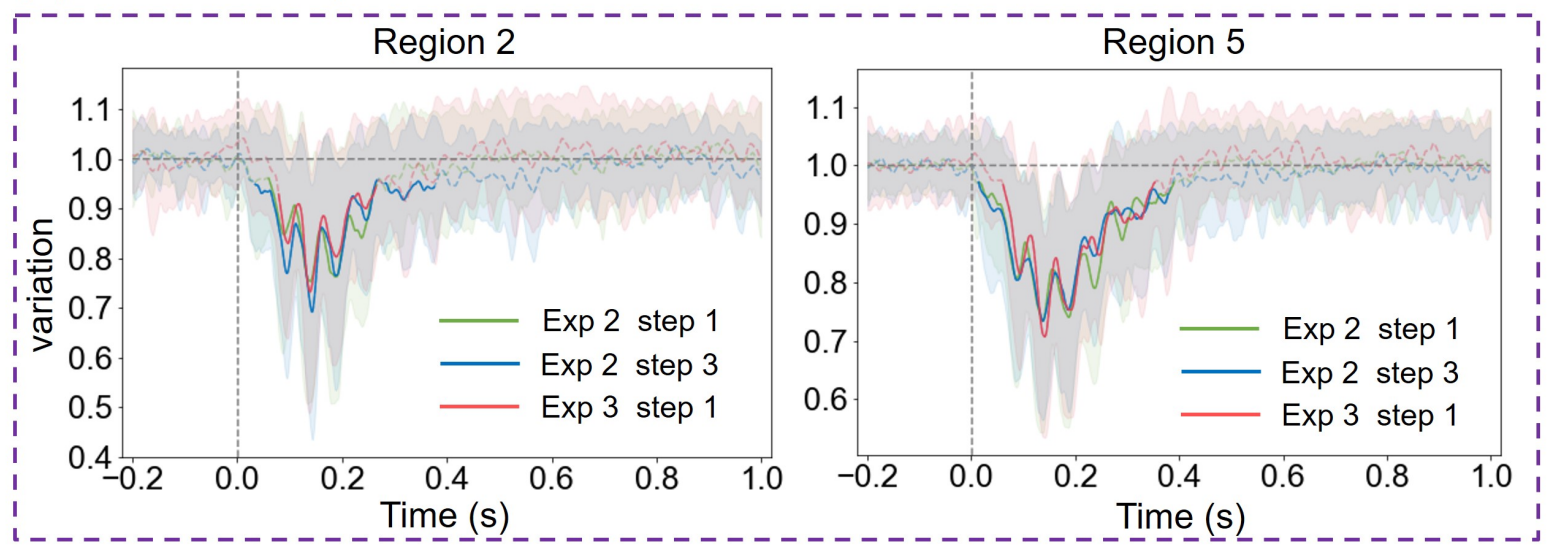

Fig. 6 EEG variations in pre-influence judgement state can predict behavioral change. (A) EEG electrode in 6 regions of interest (ROIs). The 63 electrodes were grouped into six regions. We included the electrodes in the border of two regions in both regions. (B) Some representative epochs of the EEG temporal variation in Experiment 2 and Experiment 3, region 2 (left) and region 5 (right). The green, blue, and red lines are the EEG variation calculated by the first time the face was presented in Experiment 2, the third time the face was presented in Experiment 2, and first time the face was presented in Experiment 3, respectively. The shaded areas represent the standard deviation. Dashed lines indicate that the variation is not significantly different from the baseline, and solid lines indicate that the variation is significantly different from the baseline. (C) The mean EEG variations in Experiment 2 step 1 - step 3 can predict the behavior change from pre-influence to post-influence. The left side and right side are the mean EEG variations of region 2 and region 5, respectively. The yellow dots are the subjects who were most negatively influenced. The blue dots are the subjects with little or no negative impact. Significance levels (T-test) are indicated by asterisks: $* P \leq 0.05 ; * * \leq 0.01$. 


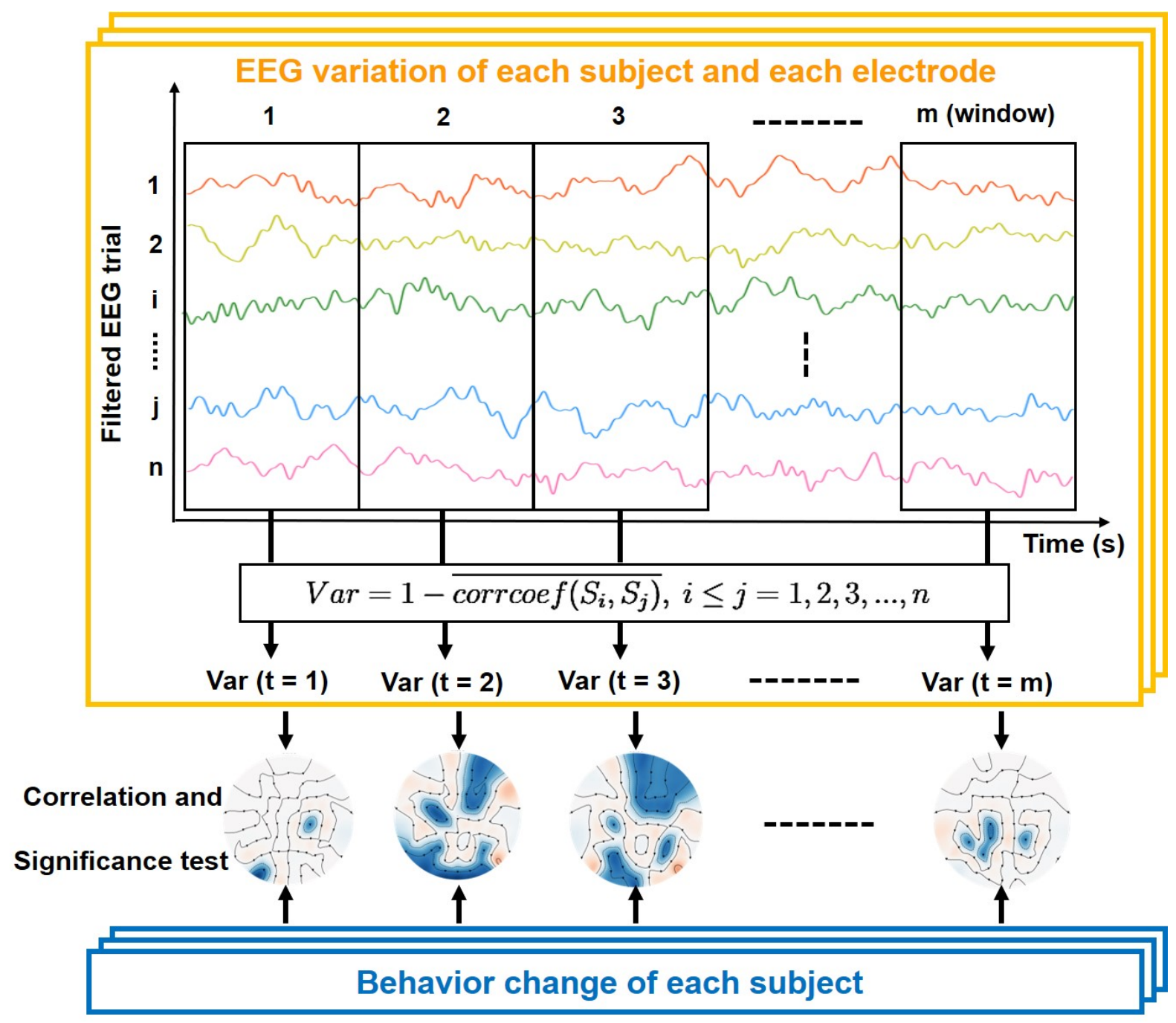

Fig. 7 The pipeline for calculating EEG temporal variation and correlating with behavioral data. The yellow box contains the definition of temporal variation. The time series are EEG signals for all trials of each subject and each electrode. We used a time window to segment EEG epoch time series. Within each time window, we calculated the similarity matrix of all trials by Pearson correlation, and averaged the lower triangle of the matrix to obtain the average EEG similarity. Next, the average EEG variation was obtained by subtracting one from the average EEG similarity. We then calculated the Pearson correlation between EEG variation and behavioral changes of all subjects. We performed statistical significant tests to the resulting correlations, and retained the correlation results with $P<0.05$. 